\title{
Factors governing one-trial contextual conditioning
}

\author{
MICHAEL S. FANSELOW \\ University of California, Los Angeles, California
}

\begin{abstract}
When a rat was placed in a chamber and shortly thereafter received a single footshock, it showed conditional freezing upon re-exposure to that chamber but not a different one (Experiment 1). Experiments 2-4 showed that the probability of this freezing decreased linearly with decreases in the delay between placement in the chamber and shock delivery. With very short delays (e.g., less than $27 \mathrm{sec}$ ), there was no freezing. Experiments 2 and 3 demonstrated that a 2 -min preexposure to the chamber, $24 \mathrm{~h}$ prior to shock delivery, reduced the minimum delay necessary to successfully condition freezing. Experiment 4 demonstrated that shorter delays were successful in conditioning freezing if a salient tone was a component of the contextual stimulus. The changes in freezing caused by delay interval and preexposure did not simply reflect the total time in the context, suggesting that there may be two requirements that place temporal restrictions on the conditioning of the freezing response. One is satisfied by sufficient exposure, whether or not that exposure is contiguous with shock. The second requirement is for a small amount of context exposure that is contiguous with shock.
\end{abstract}

If a rat receives a single aversive electric shock shortly after it is placed in a distinctive environment, the rat will exhibit a freezing response in that environment (see, e.g., Fanselow, 1980). This response is clearly a conditional response (CR) rapidly conditioned to the prevailing contextual cues. There is an important boundary condition on this finding; there must be a delay between placement in the chamber and shock delivery. If shock is delivered simultaneously with placement, no freezing is found (R. J. Blanchard, Fukunaga, \& D. C. Blanchard, 1976; Fanselow, 1986). Freezing increases linearly with increasing placement-shock delays up to at least 2 min (Fanselow, 1986). This boundary condition on postshock freezing has been termed the immediate shock freezing deficit (ISD), and it is caused by a failure of the animal to associate the contextual stimuli present at the time of shock with the shock (Fanselow, 1986). The purpose of the present experiments was to further characterize the ISD and, in particular, to explore some factors that might reduce it.

\section{EXPERIMENT 1}

One line of evidence indicating that freezing is a CR to contextual cues associated with shock is that changing the contextual stimuli between shock and testing reduces freezing in both delayed and immediate tests. Although there is a plethora of demonstrations of this context shift effect (e.g., R. J. Blanchard \& D. C. Blanchard, 1969; Bolles \& Collier, 1976; Fanselow, 1980, 1981, 1984;

This research was funded by National Institute of Mental Health Grant MH39786. Correspondence should be addressed to M. S. Fanselow, Department of Psychology, University of California, Los Angeles, CA 90024-1563.
Fanselow \& Baackes, 1982), the two studies in which the ISD was reported did not contain this manipulation. Since the parameters used to demonstrate the ISD were somewhat different-for example, only a single long shock $(2 \mathrm{sec})$ rather than multiple brief shocks $(3 \times 0.75 \mathrm{sec})-$ Experiment 1 provided a demonstration that context shifts do indeed reduce freezing when such unconditional stimulus (US) delivery parameters are used. Delayed as opposed to immediate shock was examined, because with immediate shock there is no freezing to attenuate with a context shift.

\section{Method}

Subjects. The subjects were 24 female rats derived from LongEvans stock purchased from Blue Spruce Farms (Altamont, NY). The rats were group-housed until 10 days before the experiment, when they were housed individually. During those 10 preexperimental days, the rats were adapted to handling and transport to the laboratory. At the start of the experiment, they weighed between 260 and $310 \mathrm{~g}$ and were 100 days old. Experimental procedures were conducted during the lighted portion of a $14: 10$-h light:dark cycle. The rats always had ad-lib access to water and rat chow.

Apparatus. The apparatus consisted of two different observation chambers, each of which was located in a sound- and lightattenuating chest. Clear acrylic plastic double-paned windows $(30 \times 30 \mathrm{~cm})$ in the chests allowed observation.

The test chamber was $23.5 \times 29 \times 19.5 \mathrm{~cm}$. The $23.5-\mathrm{cm}$ walls and ceiling were made of transparent acrylic plastic. The $29-\mathrm{cm}$ walls were stainless steel. Operant manipulanda were on one steel wall but were not used in these experiments. The rear plastic wall had a 15.5-cm-diameter speaker mounted at its center. Several small holes were drilled in the section of plastic that covered the speaker. These speakers were not used in this experiment. A 7.5-W (120-V) red light bulb, centered $12.5 \mathrm{~cm}$ above the ceiling of the chamber, provided illumination. Background noise $(65 \mathrm{~dB}$, A scale) was provided by ventilation fans. The grid floor was made up of 18 stainless steel rods $(2.5 \mathrm{~mm}$ in diameter $)$ spaced $1.25 \mathrm{~cm}$ center to center. Prior to each placement of a subject in it, the test cham- 
ber was cleaned with an ammonium hydroxide solution $(5 \%$ in tap water)

The different chamber was a $30.3-\mathrm{cm}$ cube. It was constructed of opaque white acrylic plastic, except for the ceiling and front wall, which were clear. The chamber was illuminated by two white lights ( 7.5 and $15 \mathrm{~W}$ ) mounted on the ceiling of the sound-attenuating chest directly over the chamber. The fans of the sound-attenuating chest were left off when a rat was in this box. This chamber was cleaned with a $2 \%$ acetic acid and tap water solution. It also had a grid floor composed of 18 brass rods ( $3 \mathrm{~mm}$ in diameter) spaced $1.5 \mathrm{~cm}$ apart.

The floors of each of the chambers were wired to a Grason-Stadler shock generator/scrambler with an output to each rod that provided a $2.0-\mathrm{sec}$, nominally $1-\mathrm{mA}$ shock.

Procedure. The rats were equally divided into three groups, in a random manner. On Day 1 of the experiment, two groups of rats were individually placed in the test chamber, and 2 min later, the rats in one of these groups (same-shock) received a single shock. Nothing happened to the other group (same-no-shock). The third group was placed in the different chamber, and 2 min later they received shock (different-shock). All rats were returned to the home cage $2.5 \mathrm{~min}$ after placement (i.e., $30 \mathrm{sec}$ after shock). On the next day, all the rats were placed in the test box for an 8-min observation period. Observation was carried out according to a timesampling procedure. Every $8 \mathrm{sec}$, each rat was judged as either freezing or active during the test, this judgment of the behavior being made at the instant that the sample was taken. Freezing was defined as the absence of visible movement, except for the minor movements required by respiration. All other behavior was considered active. The observer was blind to the previous treatment of the animal.

\section{Results and Discussion}

The design of this experiment as well as the other studies in this series meant that there would be certain groups that froze; others would evidence no freezing. This means no variance in some groups, as well as heterogeneity of within-group variance between the various conditions. Therefore, nonparametric statistics were used in all experiments. An initial overall test was made with a Kruskal-Wallis analysis followed by pairwise contrasts using the Mann-Whitney test.

The median group data are presented in Figure 1. There was an overall difference between groups $[H(2)=9.62$, $p<.05]$. The rats tested in the chamber in which they were shocked froze more than the rats that were shocked

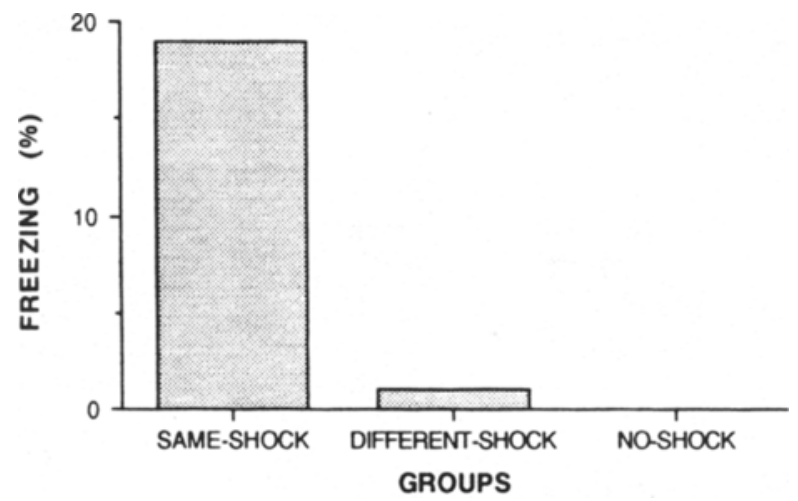

Figure 1. The median percentage of samples scored as freezing for the three groups of Experiment 1. in a different chamber $[U(8,8)=11, p<.05]$. Indeed the different-shock animals did not differ reliably from the no-shock animals $[U(8,8)=21]$, which essentially did not freeze. As in several previous studies, these data indicate that freezing is a $\mathrm{CR}$ to the apparatus cues prevalent at the time of shock.

\section{EXPERIMENT 2}

Experiment 1 showed that a 2 -min exposure to the environment immediately before shock resulted in significant conditioning to contextual cues. In previous work on the ISD, I found that if this exposure is temporally discontiguous from shock, little freezing occurs (Fanselow, 1986). In other words, preexposure does not alleviate the ISD. In those studies, the intervals between removal from the chamber and shock ranged from $15 \mathrm{~min}$ to $24 \mathrm{~h}$. Although none of the preexposed groups differed from noshock controls, mean group freezing in the preexposed rats varied from $1 \%$ to $7 \%$. In no-shock controls, the mean freezing was $0 \%$. Thus, it is possible that preexposure had an effect on the ISD that my test procedure was not sensitive enough to detect. The ISD is a graded phenomenon in that freezing increases linearly with delays of 0-81 sec between chamber placement and shock. Perhaps testing preexposed animals with a 0-sec delay was too stringent a test. Therefore, in Experiment 2, I examined the temporal delay gradient between placement in an observation chamber and shock in a manner similar to the previous procedure (Fanselow, 1986, Experiment 2). This function was compared for animals that had received a 2-min preexposure to the chamber $24 \mathrm{~h}$ earlier and those that had no such preexposure. This allowed comparison of preexposed and nonpreexposed animals at several delays to see whether there is some minimal delay interval at which preexposure results in an associative benefit. As in the first experiment, tests of freezing were conducted $24 \mathrm{~h}$ after shock.

\section{Method}

Subjects and Apparatus. The subjects were 78 adult female rats born and raised from Charles River Sprague-Dawley stock. Preexperimental treatment was identical to that of the first experiment. The test chamber of Experiment 1 was used.

Procedure. On Day 1, a random half of the animals were removed from their home cages, transported to the laboratory, placed in the chamber for $2 \mathrm{~min}$, and then returned to the home cages. The other half were transported to the laboratory but then immediately returned to the home cages.

On Day 2, all the animals were placed in an observation chamber. For animals that had been preexposed the day before, this was the same chamber that they had been exposed to earlier. Shock was delivered $1,3,9,27$, or $81 \mathrm{sec}$ after placement in the chamber. This assignment was determined randomly, except that it was balanced so as to be equated with the preexposure condition. All groups consisted of 8 subjects, except for the nonpreexposed 9-sec delay group and the nonpreexposed 81 -sec group. Those groups had 7 subjects each. All rats were removed from the chamber $30 \mathrm{sec}$ after shock termination and returned to their home cages.

On Day 3, the rats were returned to the chambers that they had been exposed to the day before and were left there, undisturbed, for a 5-min test. Freezing was again analyzed with nonparametric 
statistics. Since I was looking to see whether a benefit of preexposure would occur at any of the intervals, the a priori planned comparisons were pairwise comparisons between preexposed and nonpreexposed rats at each interval. These were made with Mann-Whitney tests when the overall Kruskal-Wallis analysis proved reliable.

\section{Results and Discussion}

The median percentages of samples scored as freezing for each group are presented in Figure 2 . As can be seen in the figure, freezing increased as delay increased, replicating earlier work done on this temporal function.

The overall Kruskal-Wallis analysis indicated a difference between groups $[H(9)=31.91, p<.01]$. Contextual preexposure reliably increased freezing at the 9-sec delay interval $[U(8,7)=9.5, p<.05]$. However, the preexposed and nonpreexposed animals did not differ from each other at any other interval.

It should be noted that the nonpreexposed controls were merely handled, rather than exposed to a different context. In subsequent research, we have determined that 2 min of preexposure to a different context immediately before shock does not alleviate the ISD. Therefore, while the present experiments do not conclusively prove that the preexposure must be to the shock context, that condition seems quite likely.

Earlier, I reported that contextual preexposure does not overcome the immediate (i.e., a 0-sec delay) shock freezing deficit (Fanselow, 1986). The present results extend those findings to delays of 1 and $3 \mathrm{sec}$. However, preexposure facilitated conditional freezing if the delay between contextual placement and shock was $9 \mathrm{sec}$. With longer delays, preexposure lost its effect. This suggests that the effects of preexposure are not just a general facilitation of freezing but rather a reflection of a more specific attenuation of the ISD.

Note that the changes in freezing caused by delay interval and preexposure do not simply reflect cumulative time in the context. For example, the preexposed 1-sec delay animals spent a total of $121 \mathrm{sec}$ in the chamber prior to shock but froze reliably less than the nonpreexposed $81-\sec$ delay animals $[U(8,7)=4, p<.01]$. This pat-

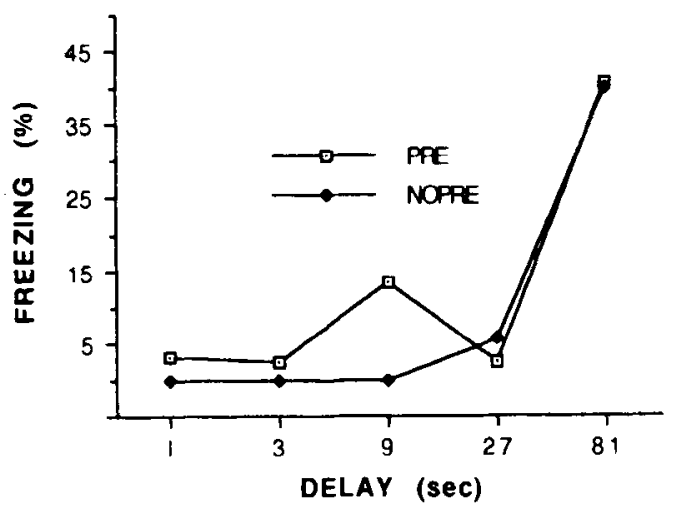

Figure 2. The median percentage of samples scored as freezing during the test session of Experiment 2, as a function of placement shock delay and context preexposure.

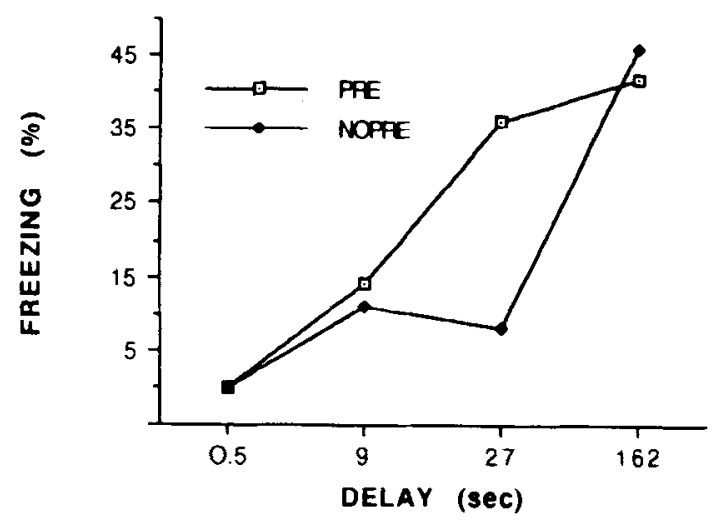

Figure 3. The median percentage of samples scored as freezing during the test session of Experiment 3, as a function of placementshock delay and context preexposure.

tern of results suggests that two factors may place temporal restrictions on the conditioning of the freezing response. One is satisfied by sufficient exposure, whether or not that exposure is contiguous with shock, and a second requires a small amount of contiguous exposure with shock.

\section{EXPERIMENT 3}

Experiment 3 was intended to replicate the findings of the second experiment. A different strain of rats was used to increase the generality of the findings.

\section{Method}

Subjects. Sixty adult female rats of Long-Evans descent served as subjects. These rats were bred from Blue Spruce Farms stock. All treatments of these rats were similar to those in the earlier experiments.

Apparatus. The equipment was that of Experiment 2.

Procedure. The procedure was identical to that in Experiment 2, with the exception that delay intervals of $0.5,9,27$, and $162 \mathrm{sec}$ were used. The longer interval was chosen in the hope of finding a nonmonotonic relationship between delay and freezing that might be expected on the basis of latent inhibition. There were 8 subjects in each of the groups that had 9-and 27-sec delay intervals. There were 7 subjects in each of the other groups. The same blind observation procedure was used.

\section{Results and Discussion}

The data, presented in Figure 3, were analyzed in the same manner as in the earlier experiments. An overall Kruskal-Wallis analysis indicated a reliable difference between groups $[H(7)=34.64, p<.01]$. Contrasts made with the Mann-Whitney test indicated that the preexposed and nonpreexposed groups differed only at the 27 -sec interval $[U(8,8)=13, p=.05]$.

The pattern of results is generally consistent with that in Experiment 2, replicating and extending those findings to another strain of rat. Freezing increased monotonically with delay. Again, the effects of preexposure were manifest only at an intermediate delay interval, not short or long intervals. One difference between the two experiments 
was that preexposure's effects were manifest at $27 \mathrm{sec}$ but not $9 \mathrm{sec}$ in Experiment 3, whereas the reverse was true of Experiment 2. But because between-experiment comparisons are not reliable, it would be premature to conclude that this represents a strain difference.

\section{EXPERIMENT 4}

The ISD may be viewed as being somewhat similar to the simultaneous conditioning deficit suggested by traditional Pavlovian theory. For example, Hilgard and Marquis (1940) state that "the procedure of conditioning in which the onset of conditioned and unconditioned stimuli is coincident ... is ordinarily an unfavorable relationship for conditioning" (p. 351). However, several reasons suggest that this simultaneous conditioning account of the ISD is inadequate. First, within traditional Pavlovian theory, it is usually thought that a delay of even a fraction of a second between the conditional stimulus (CS) and US is sufficient to result in strong conditioning (Hilgard \& Marquis, 1940, p. 44; Pavlov, 1927, p. 88), but as was seen in Experiments 2 and 3, the ISD is still apparent with delays of several seconds. Second, an explanation of the ISD in terms of simultaneous conditioning does not explain why preexposure can overcome the deficit at intermediate intervals. Third, the simultaneous conditioning deficit is more a description of a result than an explanation of that result. In this regard, it is quite possible that any explanation of the ISD would also provide an explanation of the simultaneous conditioning deficit.

In addition, more recent work in Pavlovian conditioning suggests that a delay between CS onset and US onset is not necessary for the production of strong associations (e.g., Burkhardt \& Ayres, 1978; Rescorla, 1980; Shurtleff \& Ayres, 1981). A finding by Ayres and his colleagues (Ayres, Haddad, \& Albert, 1987) is of special relevance to the phenomenon under consideration here. These researchers found that a single backward pairing of an auditory CS and a shock US was sufficient for the conditioning of the freezing response to the CS. At first glance, the Ayres et al. finding of conditional freezing with a single backward conditioning trial might appear inconsistent with the ISD. This is because one-trial backward conditioning was successful (Ayres et al.), whereas one-trial simultaneous conditioning was not (the ISD). One difference between the two sets of studies is that the ISD was found by using contextual cues as CSs, whereas Ayres' group used more traditional discrete auditory cues as CSs. This points to the possibility that the cause of the associative deficit responsible for the ISD resides in some inherent difference between these two types of CSs. One potentially important difference is that a contextual CS is composed of a complex array of stimuli, none of which is particularly salient in comparison with the more unidimensional and salient discrete CS used by Ayres et al. Therefore, in Experiment 4, the effects of adding a simple salient cue (tone) on the delay function were examined.

\section{Method}

Subjects. The subjects were 40 Long-Evans rats similar to those in Experiments 1 and 3

Apparatus. The apparatus was that of Experiment 2. However. a $10-\mathrm{kHz}$ tone was presented at $78 \mathrm{~dB}$ (A scale) through the speaker.

Procedure. The procedure was like that for the nonpreexposed animals of Experiments 2 and 3. However. delay intervals of 1 . 41. 54. and $162 \mathrm{sec}$ were used. The short and long intervals were chosen to set boundary conditions for the ISD. The intermediate intervals were chosen because pilot work had shown that a tone had no effect on freezing with intervals of less than $30 \mathrm{sec}$. Half of the animals at each delay interval had the tone continuously on while they were in the chamber. The tone turned on as the animals were being placed in the chamber. For the tone animals, the tone was present on both the training and the testing day. The other half of the rats never heard the tone. There were 5 subjects in each of the 8 groups

The same blind time-sampling procedure was used. In addition, a co-observer who was also uninformed about the rats' group assignments simultaneously observed a portion of the animals.

\section{Results and Discussion}

The two observers showed high interobserver reliability for the dependent measure $[r(7)=.99]$, with interobserver agreement on $97 \%$ of the samples. Only the data of the author are reported.

The data are presented in Figure 4. Statistical analysis was done as in the previous experiments. An overall Kruskal-Wallis test of significance proved reliable $[H(7)=25.59, p<.01]$. Mann-Whitney contrasts found that the tone and no-tone groups differed reliably only at the 41-sec interval $[U(5,5)=3, p<.05$; one-tailed]. The trend at $54 \sec$ was not reliable $[U(5,5)=8, p>.1]$.

These data suggest that there is a difference when a CS consists of contextual cues alone, as opposed to contextual cues compounded with a salient element like the tone used here. The presence of such a distinctive element seems to facilitate association formation. This suggests that one important difference between the studies of Ayres et al. (1987) on one-trial backward conditioning of freezing and the ISD studies consists in the nature of the target stimulus.

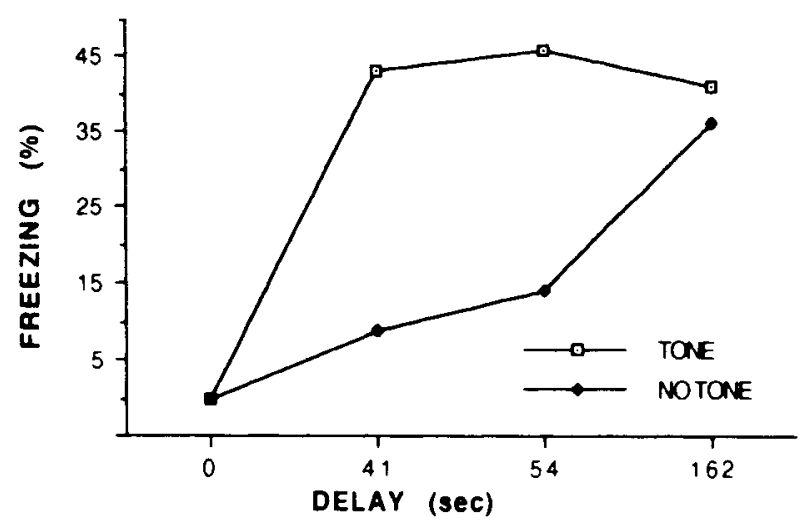

Figure 4. The median percentage of samples scored as freezing during the test session of Experiment 4 , as a function of placement shock delay and the presence of a tone. 


\section{GENERAL DISCUSSION}

Previous work (R. J. Blanchard et al., 1976; Fanselow, 1986) demonstrated that a brief period of time between the first placement in a particular context and delivery of electric footshock is necessary in order to find a postshock freezing response. When shock is delivered simultaneously with placement in the chamber, no postshock freezing is found. The failure to obtain freezing under these conditions has been termed the immediate shock freezing deficit, or ISD, and it appears to arise from a failure of the rats to form an association between the contextual cues and shock (Fanselow, 1986). The present experiments demonstrate that preexposure to the context or the presence of a salient auditory stimulus, although it does not eliminate the ISD, shortens the time period necessary to obtain detectable levels of postshock freezing.

A question of obvious importance is what is responsible for the associative deficit that causes the ISD. Earlier (Fanselow, 1986), I considered and dismissed two obvious alternatives: the simultaneous conditioning deficit (see also the introduction to Experiment 4 of this paper) and the overshadowing of the context by handling cues. The same arguments made then apply to these experiments. In addition, neither of those explanations accounts for the effect of preexposure or of adding a tone stimulus.

Another possibility is that handling automatically causes a short disruption of stimulus processing. However, two different approaches have been taken to reduce the impact of handling. In my experiments, the rats were extensively adapted to handling prior to the experiment, so any detrimental effects of handling were minimized in all groups. In the R. J. Blanchard et al. (1976) experiment, the rats were not adapted to handling, but the delayedshock rats were given an additional handling just before shock. Thus, their design maximized any detrimental effects of handling, and, in addition, those effects should have been greater in the delayed-shock animals because they were handled twice before shock. It is important that both sets of studies revealed a complete loss of freezing with immediate shock, despite these different strategies. Also, when handling is interposed between shock and immediate postshock testing, handling does not affect freezing (see Fanselow, 1982, Experiment 3). Finally, a momentary disruption caused by handling provides no insight into the effects obtained with preexposure or tone in Experiments 2-4.

The ISD suggests that exposure to the CS prior to reinforcement facilitates conditioning. The most analogous effect previously reported in the conditioning literature is that of perceptual learning (Gibson \& Walk, 1956; Hall, 1980). However, several procedural requirements that are necessary to demonstrate the perceptual learning effect (see Channell \& Hall, 1981; Hall, 1980) make its application to the ISD dubious. Perceptual learning is seen after CS exposure durations far greater than those used here. For example, Chantrey (1972) used some of the briefest intervals in the perceptual learning literature and found that discrimination learning was enhanced more by $100 \mathrm{~min}$ of exposure than it was by $30 \mathrm{~min}$ of exposure. The effects obtained in the present experiments resulted from intervals of 2 min or less. Perceptual learning does not occur when the exposure occurs in the conditioning context, yet the ISD is specifically related to exposure to the conditioning context. Finally, the perceptual learning effect is relevant to the rapidity of discrimination formation between two preexposed stimuli (e.g., Bateson \& Chantrey, 1972), but the ISD requires no specific discrimination. One might argue that in examining freezing in response to a context, one is really examining a discrimination between the test context and the home cage. However, if this analogy is made, then the preexposure manipulation used in Experiments 2 and 3 is really preexposure to both discriminanda in comparison with preexposure to only one discriminandum (the $S-$, i.e., the home cage) for the controls. Since perceptual learning research has generally resulted in better discrimination when only one of the discriminanda is preexposed (e.g., Chantrey, 1972), the pattern obtained here is opposite to those data. Thus the ISD should not be thought of as an instance of perceptual learning as it is defined in that literature. In a general sense, it is certainly possible that some sort of perceptual learning is involved in the ISD (see below), but the issues raised by the literature on the perceptual learning effect and the procedures used to investigate it do not seem readily applicable to the ISD.

A speculative explanation of the ISD can be developed out of Pavlov's conceptualization of a dynamic stereotype (Pavlov, 1955, 1962). Pavlov (1962) suggested that when an animal is confronted with a novel, complex array of stimuli, its cortical activity is chaotic. Eventually, however, the effects of these stimuli "become systematized, equilibrated, and form, so to speak, a dynamic stereotype" (Pavlov, 1962, p. 124). It is conceivable that before the complex array of stimuli referred to as a contextual CS can enter into an association with a US, that stimulus array must be synthesized into just such a dynamic stereotype. This synthesis would take a period of time during which the subject was exposed to the particular stimulus array. One contributing factor to the delay gradient of the ISD may be the time necessary for this synthesis to occur. In addition, one might assume that if the dynamic stereotype was not currently active but rather was stored in memory, a brief period of re-exposure to the stimulus array would be necessary to retrieve it and make it active. A second component contributing to the delay gradient corresponds to this retrieval time. This explanation fits the present data quite well. If context exposure is contiguous with shock, then the only time needed will be that necessary to form the dynamic stereotype, for it will be active when shock occurs. Preexposure to a context allows the animal to form the stereotype, but immediate shock does not give adequate time for the previously formed stereotype to be activated prior to shock. However, in preexposed animals, only a brief reexposure, long enough to retrieve the stereotype from 
memory, is needed for association formation. In Experiments 2 and 3, it seemed that it took somewhere between 9 and $27 \mathrm{sec}$ for this reactivation to occur.

One could apply a similar logic to the effects found with the tone. Placement of a highly salient stimulus, such as a loud tone, in the stimulus array causes the activity provoked by that stimulus to dominate the dynamic stereotype. This essentially simplifies the dynamic stereotype, so that a briefer period is required for its synthesis.

There are two ways to conceptualize the process of formation of the dynamic stereotype. One is to conceive of it as neural activity in much the way that Pavlov did. Electrical activity in various brain regions does become more uniform with repeated presentations of a stimulus compound (John, 1972; Schuckman \& Battersby, 1965). Perhaps these changes in neural activity reflect the formation of this synthesis. Another approach to this process is to view the context as consisting of independent elements of a compound. When provided with sufficient exposure to the compound, the animal forms associations between its various elements (see, e.g., Rescorla \& Freberg, 1978). These within-compound associations unify the context into an associative structure capable of entering into association with the US. With the experimental procedures used here, such associative processes appear to be relatively complete by $81 \mathrm{sec}$.

The present experiments, along with others (R. J. Blanchard et al., 1976; Fanselow, 1986), indicate that preexposing a CS prior to conditioning can facilitate association formation. This contrasts with perhaps the most familiar effect of CS preexposure-its ability to retard association formation that has been termed latent inhibition (Lubow, 1973). Perhaps, as Pavlov suggested, with enough experience the dynamic stereotype becomes "little susceptible to change and resistant to new conditions and new stimulations" (1955, p. 259). Thus there may be a nonmonotonic effect of preexposure, with short durations that just allow synthesis of the dynamic stereotype facilitating association formation, but with longer durations rendering the stereotype immutable, the result being latent inhibition. In this regard, work in my laboratory (e.g., Fanselow, 1986, and unpublished observations) suggests that no further increases in postshock freezing occur with delay intervals over $3 \mathrm{~min}$. R. J. Blanchard, Dielman, and D. C. Blanchard (1968) found reduced freezing after $24 \mathrm{~h}$ of delay relative to animals with 30 min of delay. In addition, Balaz, Capra, Kasprow, and Miller (1982) found significant latent inhibition of associations between a contextual CS and a shock US following four exposures, each of which was $1 \mathrm{~h}$ long. Therefore, it appears that the facilitative effects of contextual exposure seem to approach asymptote after only a few minutes; after that, a substantial additional period of contextual exposure is needed for the retarding or latent inhibitory effects of preexposure to become manifest. Elsewhere, I (Fanselow, 1989) have confirmed this nonmonotonic pattern for total context exposure by giving rats a single shock after 1 or more separate 30 -sec exposures to a context. Freez- ing increased between 1 and 25 exposures but decreased with 125 exposures.

Above, an explanation of the ISD in terms of simultaneous conditioning was criticized because it involved empirical description more than it did an explanation of process. The dynamic stereotype account provides an explanation of poor conditioning with simultaneously presented CSs and USs in much the same way as it accounts for the ISD. Therefore, the above speculation benefits from its ability to provide a single account of the ISD, simultaneous conditioning, and latent inhibition. A simple test of this hypothesis would be to determine if preexposure affects the CS-US interval function with discrete CSs in a manner parallel to its effect on the ISD.

There are methodological and theoretical implications of the ISD. Certainly, the interval between apparatus placement and training must be taken into account in the design of conditioning procedures, especially of those used to examine one-trial conditioning. For example, in onetrial passive avoidance, rats are given a shock shortly after they enter a novel compartment. The ISD suggests that conditioning to the compartmental cues will be strongly influenced by the delay between entrance into the chamber and shock. Interestingly, in many experiments in which this procedure has been used, some degree of delay between entrance into the chamber and shock delivery has been incorporated.

The ISD procedure may also be a useful method for separating conditional and unconditional effects of shock. If rats are tested immediately after immediate shock, the rats will only be showing the unconditional reactions to the shock because of this associative deficit. Rats tested immediately after delayed shock would show both conditional and unconditional reactions to the shock. If the test is delayed $24 \mathrm{~h}$, delayed-shock rats should only be showing conditional responses, whereas immediate-shock animals would be appropriate controls for sensitization and pseudoconditioning. Such a design may provide a particularly useful set of control comparisons for the analysis of contextual conditioning.

\section{REFERENCES}

Ayres, J. J. B., HADDAD, C., Albert, M. (1987). One-trial excitatory backward conditioning as assessed by conditioned suppression of licking rats: Concurrent observations of lick suppression and defensive behaviors. Animal Learning \& Behavior, 15, 212-217.

Balaz, M. A., Capra, S., Kasprow, W. J., Miller, R. R. (1982). Latent inhibition of the conditioning context: Further evidence of contextual potentiation of retrieval in the absence of appreciable context-US associations. Animal Learning \& Behavior, 10, 242-248.

Bateson, P. P. G., Chantrey, D. F. (1972). Retardation of discrimination learning in monkeys and chicks previously exposed to both stimuli. Nature, 237, 173-174

Blanchard, R. J., \& Blanchard, D. C. (1969). Passive and active avoidance reactions to fear-eliciting stimuli. Joumal of Comparative Physiological Psychology, 68, 129-135

Blanchard, R. J., Dielman, T. E., \& Blanchard, D. C. (1968). Postshock crouching: Familiarity with the shock situation. Psychonomic Science, 10, 371-372

Blanchard, R. J., Fukunaga, K. K., Blanchard, D. C. (1976) 
Environmental control of defensive reactions to footshock. Bulletin of the Psychonomic Society, 8, 129-130.

Bolles, R. C., Collier, A. C. (1976). The effect of predictive cues on freezing in rats. Animal Learning \& Behavior, 4, 6-8.

Burkhardt, P. E., AYres, J. J. B. (1978). CS and US duration effects in one-trial simultaneous fear conditioning as assessed by conditioned suppression of licking rats. Animal Learning \& Behavior, 6, 225-230.

Channell, S., \& Hall, G. (1981). Facilitation and retardation of discrimination learning after exposure to the stimuli. Journal of $E x$ perimental Psychology: Animal Behavior Processes, 7, 437-446.

ChANTREY, D. F. (1972). Enhancement and retardation of discrimination learning in chicks after exposure to the discriminanda. Joumal of Comparative \& Physiological Psychology, 81, 256-261.

FANSELOW, M. S. (1980). Conditional and unconditional components of post-shock freezing. Pavlovian Journal of Biological Sciences, 15. 177-182.

FAnselow, M. S. (1981). Naloxone and Pavlovian fear conditioning. Learning \& Motivation, 12, 398-419.

FAnSELow, M. S. (1982). The postshock activity burst. Animal Learning \& Behavior, 10, 448-454.

FANSELOW, M. S. (1984). Shock-induced analgesia on the formalin test: Effects of shock severity, naloxone, hypophysectomy and associative variables. Behavioral Neuroscience, 98, 79-95.

FANSELOW, M. S. (1986). Associative vs. topographical accounts of the immediate shock freezing deficit in rats: Implications for the response selection rules governing species specific defensive reactions. Learning \& Motivation, 17, 16-39.

FANSELow, M. S. (1989). The adaptive function of conditioned defensive behavior: An ecological approach to Pavlovian stimulus substitution theory. In R. J. Blanchard, P. F. Brain, D. C. Blanchard, \& S. Parmigiani (Eds.), Ethoexperimental approaches to the study of behavior (pp. 151-166). Dordrecht, The Netherlands: Kluwer.

FANSElow, M. S., BaACKES, M. P. (1982). Conditioned fear-induced opiate analgesia on the formalin test: Evidence for two aversive motivational systems. Learning \& Motivation, 13, 200-221.

Gibson, E. J., \& WALK, R. D. (1956). The effect of prolonged exposure to visually presented patterns on learning to discriminate them. Journal of Comparative \& Physiological Psychology, 49, 239-242.

HALL, G. (1980). Exposure learning in animals. Psychological Bulletin, 88, 535-550.

Hilgard, E. R., \& Marquis, D. G. (1940). Conditioning and learning. New York: Appleton-Century-Crofts.

JoHN, E. R. (1972). Switchboard versus statistical theories of learning and memory. Science, 177, 850-864.

LuBow, R. E. (1973). Latent inhibition. Psychological Bulletin, 79, 398-407.

Pavlov, I. P. (1927). Conditioned reflexes. New York: Dover.

Pavlov, I. P. (1955). Selected works. Moscow: Foreign Languages Publishing House.

Pavlov, I. P. (1962). Essays in psychology and psychiatry. New York: Citadel.

ResCorla, R. A. (1980). Simultaneous and successive associations in sensory preconditioning. Journal of Experimental Psychology: Animal Behavior Processes, 6, 207-216.

Rescorla, R. A., \& Freberg, L. (1978). The extinction of withincompound flavor associations. Learning \& Motivation, 9, 411-427.

Schuckman, H., \& Battersby, W. S. (1965). Frequency specific mechanisms in learning: 1 . Occipital activity during sensory preconditioning. Electroencephalography \& Clinical Neurophysiology, 18, 45-55.

SHURTLEFF, D., \& AYRES, J. J. B. (1981). One-trial backward excitatory fear conditioning in rats: Acquisition, retention, extinction, and spontaneous recovery. Animal Learning \& Behavior, 9, 65-74.

(Manuscript received February 27, 1990; revision accepted for publication June 6, 1990.) 